\title{
Facile Synthesis of Various 1-Azabicyclo[n.4.0]alkanes via Beckmann Rearrangement/Allylsilane Cyclization
}

\author{
Kyung-Tae Kang, Tae Myung Sung, Hyun Chul Jung, and Jong Gun Lee \\ Department of Chemistry and Chemistry Institute for Functional Haterials, Pusan National Lniversitv, Busan 609-735, Korea \\ E-mail: hythangapusan.ackr \\ Recerved June 13,2008
}

Key Words : Allylsilane. Beckmann rearrangement. 3-Stannyl-2-(silỵlmethyl)propene. N-Heterocycle

The carbon-carbon bond formation by the reaction of allylsilanes with electrophiles has been widely used in organic synthesis. ${ }^{3}$ Particulary. intrantolecular cyclization of allysilanes bearing an electroplilic terninus has an extensive application for the highly regio- and stereo-selective synthesis of various ring compounds.

The bismetallic reagent 3-stannyl-2-(silylntetlyl)propene $1^{2}$ should be a versatile conjunctive reagent since the allylstannane and the allylsilane moieties of 1 could be manipulated sequentially and in a controlled manner. ${ }^{3}$ Indeed, the allylstannane moiety of 1 selectively react with an aldehyde to yield hydroxy ally lsilane $2^{4}$ The reactions of $\mathbf{2}$ with either vinyl ethers or $\alpha$-halo ethers give acetals which are subsequently cyclized to afford 2,6-cis-disubstituted-4-methylenetetrahydropy rans 3 .

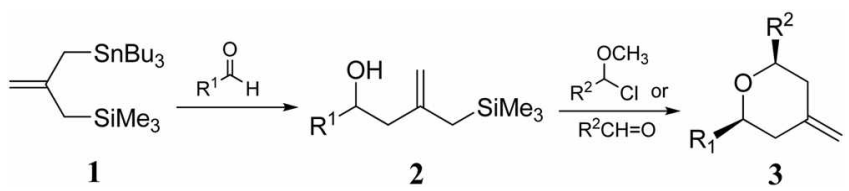

Enantioselective synthesis of the tetrahydropyrans 3 was achieved by using the hydroxy allylsilanses 2 generated from the catalytic asymmetric allylation of 1 with aldehydes. ${ }^{\circ}$ This annulation reaction enabled an efficient synthesis of the biologically active tetrahydropyran natural products. ${ }^{7}$ Various 2.6-disubstituted 4-methy lenepiperidines were also prepared in one-pot by the sequential reactions of aldinines with bismetallic reagent 1 followed by aldehydes. ${ }^{8}$

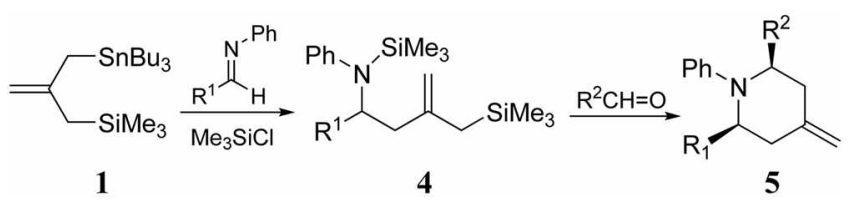

We described herein the synthesis of 1-azabicyclo[n.4.0]alkanes using bismetallic reagent 1 . Reaction of $\alpha$-tetralone oxime mesy late 6 a with 2 equivalents of trimethylaluminum resulted in the fomation of cyclic ketimine 8a. Methylation of intermediate iminocarbocation $7 \mathbf{a}$. which was generated from the organoaluminum-promoted Beckmann rearrangement of oxime mesylate $6 \mathbf{a}$. with trimethylaluminum afforded cyclic imine $\mathbf{8} \mathbf{a}$. Allylation of cyclic ketimine $\mathbf{8 a}$ with allỵllithium 9 gave cyclic amino allỵlsilane 10a in good yield. ${ }^{10} 2$-(Trimethylsilylmethyl)allyllithium 9 was generated by treating bismetallic reagent 1 with methyllithium.

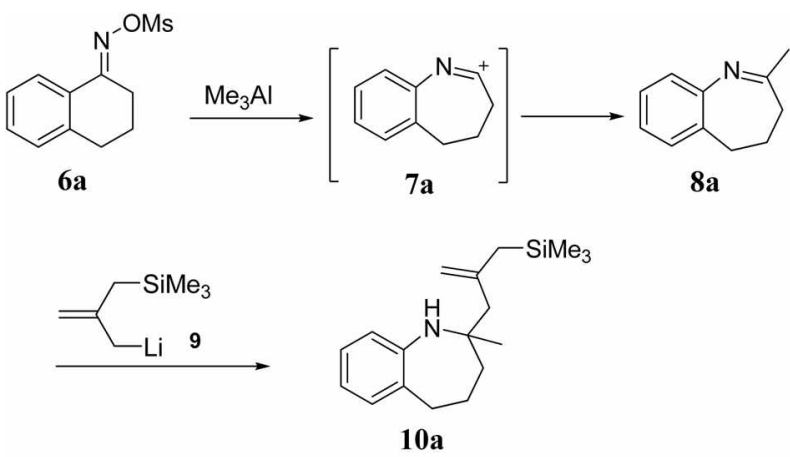

This easy and one-pot reaction has a wide generality. Other synthetic examples of this type are given in Table 1. It permits the introduction of an allylsilane moiety into a substrate with a simultaneous ring expansion.

In the synthesis of ketimines $10 \mathrm{a}$ and $10 \mathrm{~h}$, rigorous regioselectivities were observed. The phenyl group anti to departing mesylate group migrated preferentially. ${ }^{9}$ For some reasons, this process did not work for all the oxime mesylated tested. For example. reaction of 1 -indanone oxime mesylate $6 \mathbf{j}$ with trimethylaluminum and followed allyllithium 9 under standard reaction condition gave only dially lation product $11 \mathrm{in}$ low yield. ${ }^{11}$ Even with larger excess of trimethylaluminum (4 equiv) and after prolonged reaction time, the expected monoallylation product 10i was not produced. It is not clear why such anomalous behavior was observed for l-indanone oxime mesylate only.

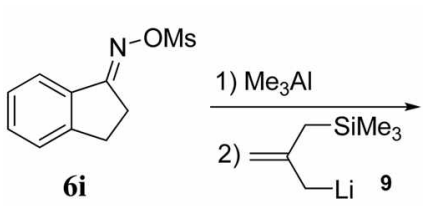<smiles>C=C(CC)CC1(CC(=C)CC)CCc2ccccc2N1</smiles>

11<smiles>C=C(CC)CC1(C)CCc2ccccc2N1</smiles>

$10 \mathrm{i}$

Mannich cyclization of ininium-vinyl and allylsilanes is to provide an attractive method for the regio-controlled production of piperidines possessing either endo- or exo- 
cyclic unsaturation. ${ }^{12}$ Cyclic amino allylsilanes 10 as their trifluoroace tate salts were treated at $40-45^{\circ} \mathrm{C}$ with 1.2 equiv. of formaldehyde in water:tetrahydrofuran $(3: 1)$ to give azabicyclic compounds 13 . $^{13}$

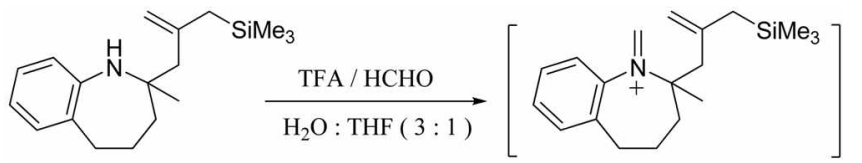

10a

12a

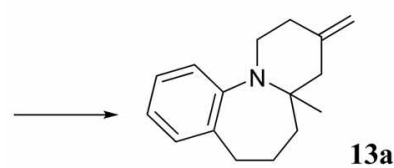

13a

Table 1. Synthesis of cyclic anino allylsilanes 10 and 1 -azabicyclo[n.4.0]alkanes 13

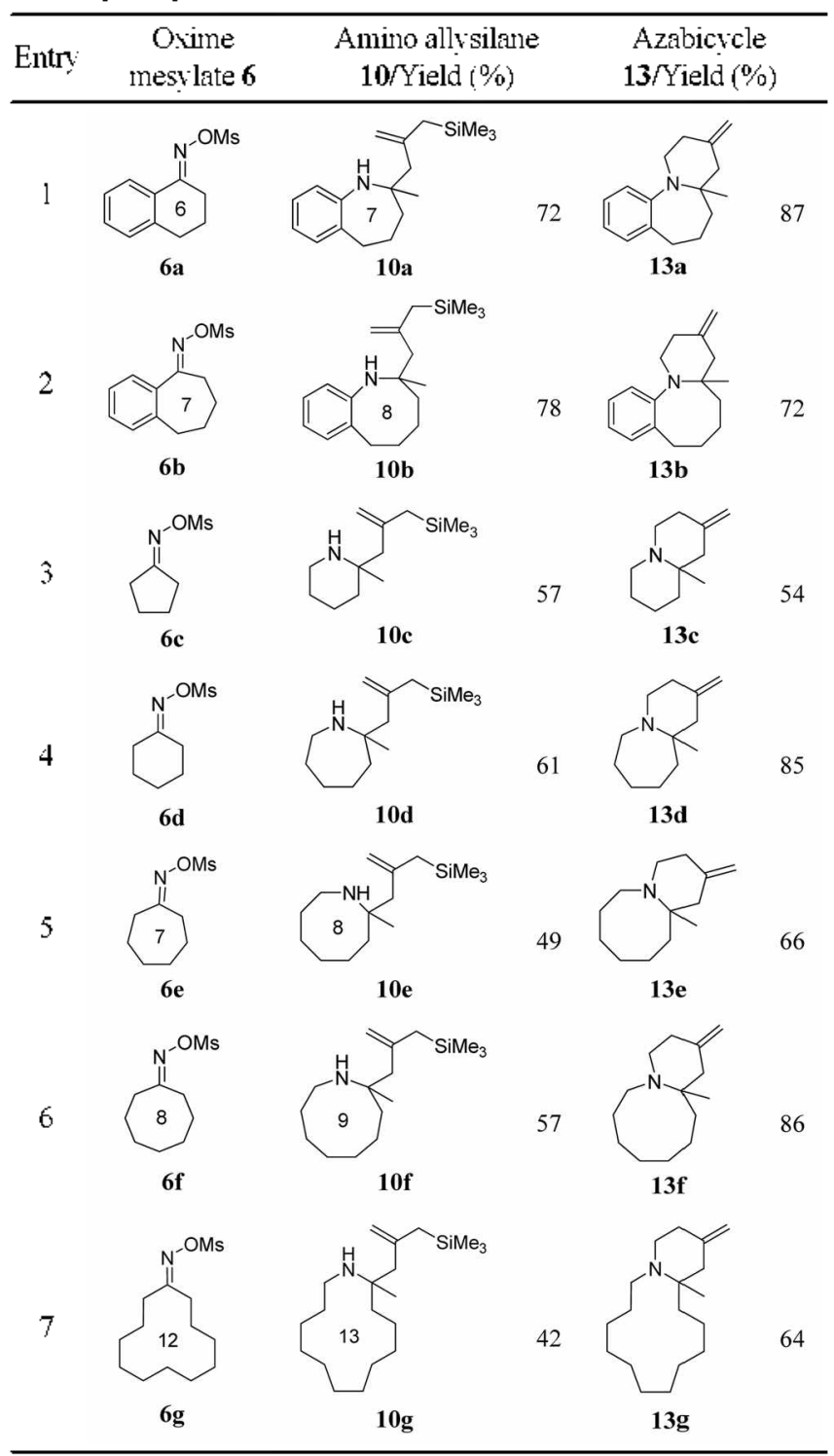

As shown be seen in Table 1. 1-azabicyclo[n.4.0]alkanes of various ring size $(n=4,5.6,7$ and 11$)$ were obtained in good yields.

The present reaction sequence, organoaluminum-promoted Beckmann rearrangement of oxime mesylate, allylation reaction with 2-(trimethylsily lmethyl)allyllithium, and Mannich reaction, provides a versatile and useful synthetic method for 1-azabicylo[n.4.0]alkanes.

Acknowledgments. This work was supported for two years by Pusan National University Research Grant.

\section{References and Notes}

I. (a) Fleming. I.: Dunogues. J.: Smithers. R. Ong React. 1989.37.57. (b) Langhopf. E.: Schinzer. D. Chem. Rev: 1995. 95. 1375. (c) Chabaud. L.: James. P.: Landais. Y. Eut J. Ong Chem. 2004. 3173.

2. (a) Kang, K.-T.: U. J. S.; Park. D. K.: Kim. J. G.; Kim. W. J. Bull. Korean Chem. Soc. 1995. 16, 464. (b) Benoit. D.: Bemand, L. Synlett 2006. 2148.

3. (a) Clive. D. L. J.: Paul. C. C.: Wang. Z. J. Org. Chem. 1997. 62. 7028. (b) Kang. K.-T: Hwang. S. S.: Kwak. W. Y: Yoon. U. C. Bull. Korem Chent. Soc. 1999. 20.801.

4. (a) Majetich. G: Mishidie. H: Zhang. Y. J. Chem. Soc. Perkin 1 1995. 453. (b) Kang, K.-T.: U. J. S.; Park. D. K.: Kim. J. G. Kwon. Y. M. Symh Conmun 1997. 27. 1173. (c) Takumea, A. Saito. H.: Nishigaichi. Y. Chen. Conmum. 1999. 1463.

5. Sung. T. M.: Kwak. W. Y.: Kang. K.-T. Bull Korem Chem. Soc. 1998. 19.862

6. (a) Yu. C.-M.; Lee. J.-Y.: So, B.; Hong. J. Angew: Chem Int Ed. 2002. 41, 161. (b) Keck. G. E.: Covel. J. A.: Schiff, T.: Yu, T. Org. Lett. $2002,+1189$.

7. (a) Keck. G. E.: Truong. A. P. Org. Lett. 2005. 7. 2153. (b) Sanchez. C. C.: Keck. G. E. Org. Lett. 2005. 7.3053

8. Kan1g. K.-T.: Kim. E. H.: Kinn. W. T.: Song. N. S.: Shin1. J. K.: Cho. B. Y. Synlett 1988.921

9. (a) Maruoka. K.: Mivazali. T.: Audo. M:- Matsumara. Y.: Sakane. S.) Hattori. K: Yamamoto, H. J. Am. Chem Soc. 1983. 105, 2831. (b) Schinzer. D.: Bo. Y. Angew: Chem. Int. Ed. Engl. 1991. 30. 687. (c) Schinzer. D.: Langhopt. E. Sintett 1994. 375.

10. 10a: 'H NMR $\delta 0.04$ (9H.s). $1.06(3 \mathrm{H} . \mathrm{s}) .1 .62-1.72(4 \mathrm{H} . \mathrm{m}) .1 .63$ (1H. d. $J=13.2 \mathrm{~Hz}), 1.74(1 \mathrm{H} . \mathrm{d} . J=13.2 \mathrm{~Hz}), 2.08(1 \mathrm{H} . \mathrm{d} . J=$ $13.0 \mathrm{~Hz}), 2.23(1 \mathrm{H} . \mathrm{d} .13 .0 \mathrm{~Hz}), 2.74(2 \mathrm{H}, \mathrm{t} . J=4.8 \mathrm{~Hz}) .3 .74(1 \mathrm{H}$. brs). $4.74(1 \mathrm{H}, \mathrm{s}), 4.81(1 \mathrm{H}, \mathrm{s}), 6.67-6.98(4 \mathrm{H}, \mathrm{m}){ }^{13} \mathrm{C}$ NMR $\delta 1.5$. 22.5. 25.5.29.5.35.5.42.7.50.6.549.111.9.121.0. 121.2. 126.4. 129.9. 134.0. 144.3. 146.2: HRMS mz 287.2080 $\left(\mathrm{C}_{18} \mathrm{H}_{2} \mathrm{uNSi}\right.$ requires 287.2071 ).

11. 11: 'H NMR $\delta 0.00(18 \mathrm{H}, \mathrm{s}), 1.61(4 \mathrm{H}, \mathrm{d}, J=13.2 \mathrm{~Hz}), 1.71(4 \mathrm{H}$. d. $J=13.2 \mathrm{~Hz}), 1.83(2 \mathrm{H}, \mathrm{t} . J=6.8 \mathrm{~Hz}) .2 .10(4 \mathrm{H} . \mathrm{d} . J=13.4 \mathrm{~Hz})$. $2.26(4 \mathrm{H} . \mathrm{d} . J=13.4 \mathrm{~Hz}) .2 .78(2 \mathrm{H} . \mathrm{t} . J=9.8 \mathrm{~Hz}), 3.90(1 \mathrm{H}, \mathrm{brs})$. 4.59 (2H.s). 4.71 (2H. s). 6.46-7.06 (4H. m): ${ }^{3} \mathrm{C}$ NMR $\delta-1.3$. 23.7. 29.4. 30.4. 46.7. 54.0. 111.4. 114.7. 116.7. 120.4. 126.7 . 129.2. 143.9. 144. $)$. HRMS mz $385.2619\left(\mathrm{C}_{23} \mathrm{H}_{39} \mathrm{NSS}_{2}\right.$ requires 385.2623 ).

12. Grieco, P. A.; Fobare, W. F. Tetrohedron Lett. 1986. 27.5067.

13. $13 \mathrm{a} .{ }^{3} \mathrm{H}$ NMR $\delta 0.97(3 \mathrm{H} . \mathrm{s}), 1.26-1.42(2 \mathrm{H}, \mathrm{m}), 1.52-1.72(1 \mathrm{H}$. m). $1.80-1.99(2 \mathrm{H} . \mathrm{m}) .2 .28-2.45(2 \mathrm{H} . \mathrm{m}) .2 .48-2.74(2 \mathrm{H} . \mathrm{m})$. $2.92-3.50$ (3H. m). 4.75 (lH. s). 4.88 (lH. s). $6.88-7.28$ (4H. m): ${ }^{13} \mathrm{C}$ NMR $\delta$ 19.2. 19.5. 30.3. 35.5. 37.7. 44.9. 46.8. 56.1. 109.6. 121.4. 122.5. 126.6. 128.2. 137.0, 145.6, 148.8: HRMS $m z$ $227.1681\left(\mathrm{C}_{16} \mathrm{H}_{21} \mathrm{~N}\right.$ requires 227.1675$)$. 